\title{
A model system for the study of human retinal angiogenesis: activation of monocytes and endothelial cells and the association with the expression of the monocarboxylate transporter type 1 (MCT-1)
}

\author{
R.M. Knott, M. Robertson, E. Muckersie, V. A. Folefac, F. E. Fairhurst, S. M. Wileman, J. V. Forrester \\ Department of Ophthalmology, University Medical Buildings, Foresterhill, Aberdeen, UK
}

\begin{abstract}
Aims/hypothesis. The growth of retinal vessels is associated with a number of disease conditions, including diabetic retinopathy and proliferative vitreo-retinopathy. In this study we describe a model of human retinal angiogenesis and show how this may be used to explain the mechanisms that are associated with the growth of new retinal vessels.

Methods. A $4 \mathrm{~mm}$ diameter disc of retinal tissue was placed within a fibrin matrix and the appearance was monitored daily by light microscopy. Immunohistochemical techniques were used for the detection of, glial fibrillary acidic protein, CD68, the Ki-67 antigen, vascular endothelial growth factor, monocarboxylate transporter type 1 and von Willebrand's factor.

Results. Vessels were evident extending from the periphery of the explant and the activation of endothelial cells was shown by immuno-peroxidase staining of paraffin embedded sections of the explants for the expression of the Ki-67 antigen, a marker of cell pro-
\end{abstract}

liferation. The expression of glial fibrillary acidic protein and von Willebrand's factor increased with duration in culture and the presence of activated macrophages or microglia or both was shown by positive immunoreactivity for CD68 and Ki-67 and were identified by day 3 . The presence of endogenous vascular endothelial growth factor and the activation of monocarboxylate transporter type 1 by vascular endothelial growth factor, showed the involvement of specific growth factors.

Conclusion/interpretation. The explant model provides evidence for the involvement of macrophages and glial fibrillary acidic protein activation in human retinal angiogenesis and for the expression of monocarboxylate transporter type 1 , which is likely to be important in the use of lactate in the hypoxic retina. [Diabetologia (1999) 42: 870-877]

Keywords Retina, angiogenesis, growth factors, ischaemia, diabetic retinopathy, monocarboxylate transporter type 1
Received: 9 December 1998 and in final revised form: 26 January 1999

Corresponding author: Dr R.M. Knott, Department of Ophthalmology, University Medical Buildings, Foresterhill, Aberdeen AB25 2ZD, UK

Abbreviations: PDR, Proliferative diabetic retinopathy; VEGF, vascular endothelial growth factor; bFGF, basic fibroblast growth factor; PDGF, platelet derived endothelial growth factor; MCT-1, monocarboxylate transporter type 1; GMEM, Glasgow's minimal essential medium; APAAP, alkaline phosphatase-antialkaline phosphatase; vWF, von Willebrand's factor; GFAP, glial fibrillary acidic protein; TBS, Tris buffered saline; H\&E, haemotoxylin and eosin; Mag., magnification.
Diabetic retinopathy is characterised at different stages of disease by an increase in vascular permeability, increased blood flow, thickening of the basement membrane, capillary occlusion, microaneurysm formation, retinal ischaemia and the appearance of new vessels (angiogenesis) [1]. The association of new vessel growth that characterises proliferative diabetic retinopathy (PDR) appears to be associated with ischaemic areas of the retina in patients with diabetes [2] which is thought to be an essential component in the angiogenic stimulus. The precise mechanism whereby activation of cell growth is achieved is, however, unclear although it is known that ischaemia 
is in itself a stimulus for changes in endothelial cells resulting in cytoplasmic swelling, protrusions into the lumen and decreased numbers of pinocytic vesicles [3]. Coincident with ischaemia is hypoxia that is known to alter the expression of specific growth factors. This includes the expression of vascular endothelial growth factor (VEGF), a potent endothelial cell mitogen [4] by retinal glial cells [5] and the increased expression of basic fibroblast growth factor (bFGF) and platelet derived endothelial cell growth factor (PDGF) by mononuclear cells [6]. The importance of $\mathrm{O}_{2}$ tension is illustrated by showing that high oxygen levels inactivate acidic and bFGF with a consequent decrease in endothelial cell proliferation [7].

The ability of endothelial cells to use monocytederived growth factors is clearly dependent upon their residence in the retinal microvasculature [6]. Evidence for the activation of endothelial cells [8] and monocytes [9-11] has been identified in association with diabetes which further supports this notion. Furthermore, there is an association between diabetes and leucocyte-mediated capillary occlusion, monocyte activation and new vessel growth in the retina of animals with diabetes [10], and hypoxia has been shown to increase leucocyte adhesion to the endothelium [12]. Increased expression of integrins $\left(\alpha_{5} \beta_{3}\right.$ and $\left.\alpha_{5} \beta_{5}\right)$ on bovine retinal endothelial cells [13] has been shown such that monocyte-endothelial cell interaction can be facilitated.

Evidence of degenerative processes in the retina is an early indicator of retinal damage and is apparent prior to evidence of retinal vascular damage $[14,15]$ including loss of colour [16] and contrast sensitivity [17]. Glial cells have an important role in retinal homeostasis [18] and have been shown to be involved in the formation of pre-retinal membranes [19] and impaired glial glutamate metabolism [15]. Another important feature of diabetic retinopathy is its association with poor glucose control [20] and the requirement to understand the glucose transporter systems of the retina $[21,22]$ which has been shown to use a facilitative glucose transporter system [23]. In the ischaemic retina, the facilitative glucose transporters are unable to respond to the generation of high concentrations of lactate via anaerobic glycolysis. This necessitates the expression of a suitable transport system to cope with the demands imposed by high lactate [24], namely the monocarboxylate transporter family of which there are six known members [25]. The expression of one of the monocarboxylate transporters, namely MCT-1, is associated with increased lactate uptake in both skeletal and heart muscle [26] and with lactate uptake in astroglial cells [27]

The requirement for a suitable model system for examining the effect of ischaemia on human retinal endothelial cell responsiveness is paramount in the study of the mechanism of retinal angiogenesis but it

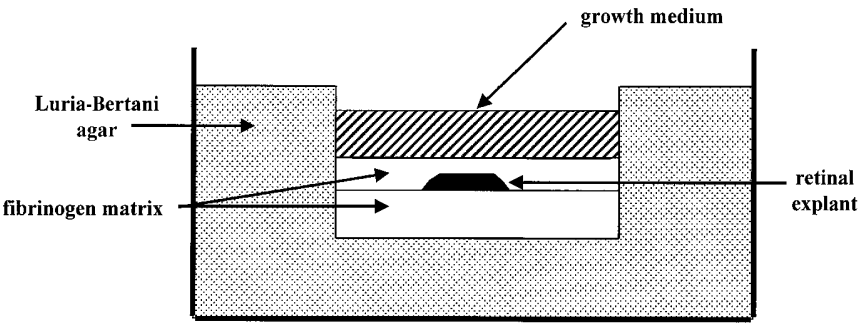

Fig. 1. Diagramatic representation of the human retinal explant model showing an outer agar mould and a central fibrin matrix. The retinal tissue is placed on top of the fibrin matrix with a further $200 \mu \mathrm{l}$ volume of fibrin matrix, followed by growth medium as specified in the Materials and methods section

is clearly not an easily attainable goal. Current models of retinal angiogenesis are restricted to animals with the inevitable caveats that are associated with their use. We have recently developed a human model of retinal angiogenesis based upon the bovine retinal explant model developed previously [28], with specific refinements. In this study we describe new vessel growth in the human retina coincident with endothelial cell and monocyte activation and increased immunoreactivity to glial fibrillary acidic protein (GFAP). We also present evidence for the expression of a monocarboxylate transporter type 1 (MCT-1) in the retinal endothelium that could be relevant to the ability of the retinal endothelium to respond to the demands of glucose metabolism and consequent lactate production in the ischaemic retina.

\section{Materials and methods}

Preparation of retinal explants. Type 1 collagen (Sigma Chemical Company, Poole, Dorset, UK) was used at concentrations of 1.25 to $2.5 \mathrm{mg} / \mathrm{ml}$ prepared in Glasgow's minimal essential medium (GMEM) supplemented with glucose $(0,5,15$ or $25 \mathrm{mmol} / \mathrm{l}) 10 \%$ fetal calf serum, $10 \mathrm{mmol} / \mathrm{l} \mathrm{NaOH}$ and $10 \mathrm{mmol} / \mathrm{l} \mathrm{HEPES}$ buffer. A volume of $300 \mu \mathrm{l}$ was placed into a $30 \mathrm{~mm}$ well of a tissue culture plate and allowed to solidify. Human eyes that had been donated for corneal transplant in Amsterdam were kindly donated from the National Ophthalmic Research Institute Eye Bank of the Netherlands, Amsterdam, after the cornea had been removed. The donors had no history of diabetes and the retinas were dissected from eyes within $48 \mathrm{~h}$ of death and spread out in a Petri dish containing HANK'S balanced salt solution. We used 17 individual retinas for the explant preparations. Each of the explants was $4 \mathrm{~mm}$ in diameter, removed with a biopsy needle and placed on top of the matrix. A fibrin matrix was prepared $(\mathrm{NaOH}$ $75 \mathrm{mmol} / \mathrm{l}, \mathrm{FCS} 2.1 \%$, thrombin $0.6 \mu / \mathrm{ml}$, D-glucose $4.7 \mathrm{mmol} / \mathrm{l}$, $1.54 \mathrm{mg} / \mathrm{ml}$ fibrin) and allowed to solidify within a Luria-Bertani agar mould in the well of a $30 \mathrm{~mm}$ tissue culture dish (Fig.1). The explants were prepared as described above and placed on top of the fibrin matrix. The explants were then overlaid with a further volume of fibrin matrix $(200 \mu \mathrm{l})$, followed by Glasgow's minimal essential medium (GMEM, Gibco BRL, Paisley, UK) containing $2.5 \%$ platelet deprived se- 
rum, supplemented with $5 \mathrm{mmol} / \mathrm{l}$ D-glucose. The explants were incubated at $37^{\circ} \mathrm{C}$ and visualised by phase contrast light microscopy from 1 to 14 days and recorded as photomicrographs. Matrix supplements included VEGF (100 ng/ml), bFGF $(50 \mathrm{ng} / \mathrm{ml})$, and a range of glucose concentrations (0-25 mmol/l).

Haemotoxylin and eosin staining of human retinal explants. Fibrin matrices containing the retinal explants were removed from the agarose mould and placed in buffered formal saline for a minimum of $4 \mathrm{~h}$, prior to embedding in paraffin wax. The blocks were then sectioned and stored at room temperature for subsequent immunohistochemical analysis. We examined 95 explants. Haematoxylin and eosin staining was carried out on each explant using standard methodology by the Department of Pathology at the University of Aberdeen.

Immunohistochemistry. The expression pattern of specific proteins in sections of 61 of the human retinal explants was determined by immunostaining using the alkaline phosphatase-anti-alkaline phosphatase (APAAP) technique [29] for von Willibrand's factor (vWF), GFAP, CD68, VEGF and monocarboxylate transporter type 1 (MCT-1). Briefly, the wax was cleared from the sections with histoclear and heated with microwaves $(800 \mathrm{~W})$ for $10 \mathrm{~min}$. The primary antibodies were diluted in $0.05 \%$ TRIS-buffered saline to 1:150 for vWF (DAKO, Cambridge, UK), and 1:100 for the GFAP (Vector Laboratories, Berkshire, UK), 1:25 for CD68 (DAKO), 1:100 for the MCT1 (Chemicon International, Harrow, UK) and 1:100 for VEGF (Insight Biotechnology, Middlesex, UK). The respective primary antibodies were added to the explant sections for $60 \mathrm{~min}$ followed by three washes in TRIS-buffered saline ( $5 \mathrm{mmol} / \mathrm{l}$ TRIS pH 7.6) (TBS). A biotinylated secondary antibody was added for a further $60 \mathrm{~min}$, washed three times in TBS and the streptavadin alkaline phosphatase was added for 30-60 min. The alkaline phosphatase substrate was then added to develop the reaction. The sections were then counterstained in haematoxylin and Scott's water and mounted. Positive staining was evident by pink staining in contrast to the negative control that was stained in the absence of primary antibody and with an IgG specific control for the respective antibodies.

Immunohistochemical detection of the Ki-67 antigen was carried out using the Mib1 antibody (Immunotech/Serotech, Oxford, UK) at a 1:100 dilution. Briefly, the staining procedures were used as outlined for APAAP staining with the following exceptions: endogenous peroxidase activity was blocked with $\mathrm{H}_{2} \mathrm{O}_{2}(0.6 \%)$ and with human serum $(10 \%)$; a biotinylated secondary antibody was added for $60 \mathrm{~min}$, followed by streptavadin horseradish peroxidase for up to $60 \mathrm{~min}$. The sections were then counter stained and mounted as described previously.

\section{Results}

Growth of retinal vessels in human retinal explants. Preliminary attempts to use a collagen matrix for the explant model as described previously [28] proved to be less efficient at sustaining vessel growth than using a fibrin matrix. When using a concentration of collagen similar to that used for the bovine retinal explant [28] no vessel spikes or changes to the periphery of the explant was evident. When the concentration was reduced to $1.25 \mathrm{mg} / \mathrm{ml}$, small vessel spikes were evident

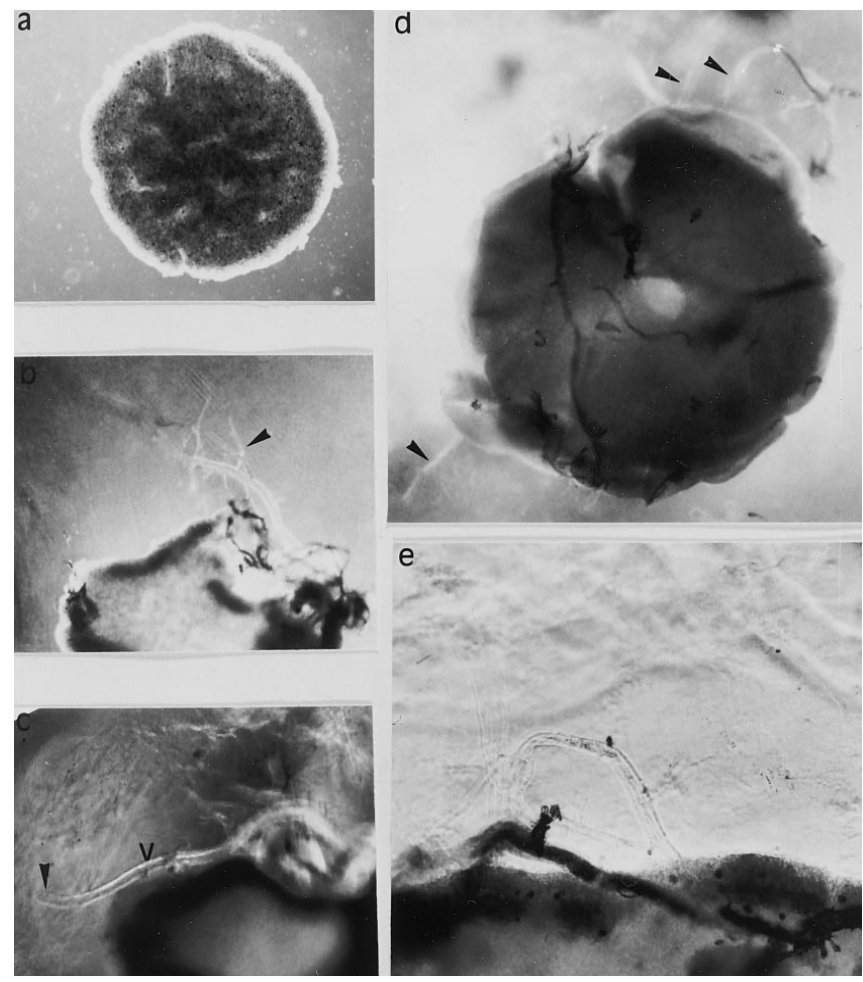

Fig. 2a-e New vessel growth from human retinal explants visualised by light microscopy (a) Retinal explant $2 \mathrm{~h}$ after placing within fibrin matrix (magnification $(\mathrm{mag}$. $) \times 7$ ) and $(\mathbf{b})$ retinal explant at day 3 (mag. $\times 9$ ). Vessels were evident extending from the retinal explant into the matrix of the gel (v).(c) Same retinal explant as for $(\mathbf{b})$ at day $6(\mathrm{mag} . \times 9)$. The branching vessel that was visible in (b) is now a complex network of vessels and this is difficult to focus on clearly. Another vessel is now evident $(\mathrm{v})$ which may have been unmasked by the retraction of the retina but shows a further area of growth as it extends away from the periphery of the explant (indicated by arrow). (d) Highly vascularised explant showing evidence of vessel growth (indicated by arrows) (mag. $\times 13$ ) after 15 days in culture. (e) Same retinal explant as for (d) showing bloodfilled vessel at edge of explant and opaque new vessels extending out from this into the surrounding matrix $(\mathrm{mag} . \times 26)$

at day 3 but these did not progress with further culture. In contrast, when using the fibrin matrix new retinal vessels were evident by day 3 as visualised by light microscopy (Fig. 2) in explants taken from 13 of the 17 eyes tested $(76 \%)$. Explants from the remaining 4 eyes $(24 \%)$ showed no evidence of new vessel growth. These donors were generally from an older age group i. e. over 75 years old and the failure to observe new vessel growth may also be due to the extended time between enucleation and sampling the retina which was beyond our control. Of the retinas that did produce explants with new vessels (13 retinas), between 83 and $100 \%$ of the explants taken from the respective retinas were found to have new vessels as determined by light microscopy. Smooth muscle cells were not associated with the new vessels as determined by a lack of immunopositivity to $\alpha$-actin (data not shown). Over a 10-day period of culture there was some retraction of 


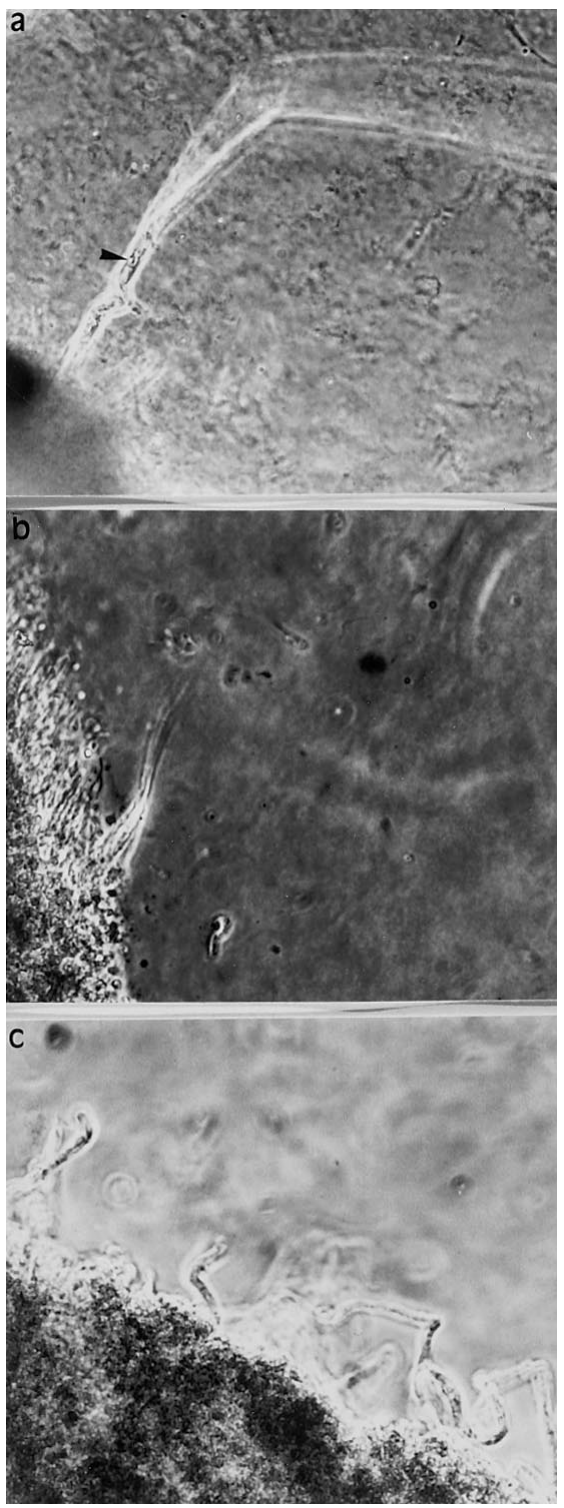

Fig.3a-c New vessel growth through the fibrin matrix from human retinal explant after 15 days culture. (a) Cells are visible within the growing vessel (mag. $\times 28$ ). (b) The same field of view is recorded as for (a) but focused on the point of origin from the explant (mag. $\times 28)$. (c) Extensive growth of vessels is evident from the edge of the explant (mag. $\times 24$ )

the explant, although at $10-20 \mu \mathrm{m}$ this was less than the distance that the vessels extended beyond the periphery of the explant which was more than $50 \mu \mathrm{m}$ (Fig.2b-e, Fig.3). Therefore the appearance of new vessels was not simply due to the retraction of the tissue to reveal pre-existing vessels. No difference in the ability of the vessels to grow was evident in $0,5,15$ or $20 \mathrm{mmol} / \mathrm{l}$ glucose.

Increased vascularity and expression of glial fibrillary acidic protein in retinal explants. We took six explants from three of the retina, and examined them after $24 \mathrm{~h}$ in culture. All of the explants examined (6/6)

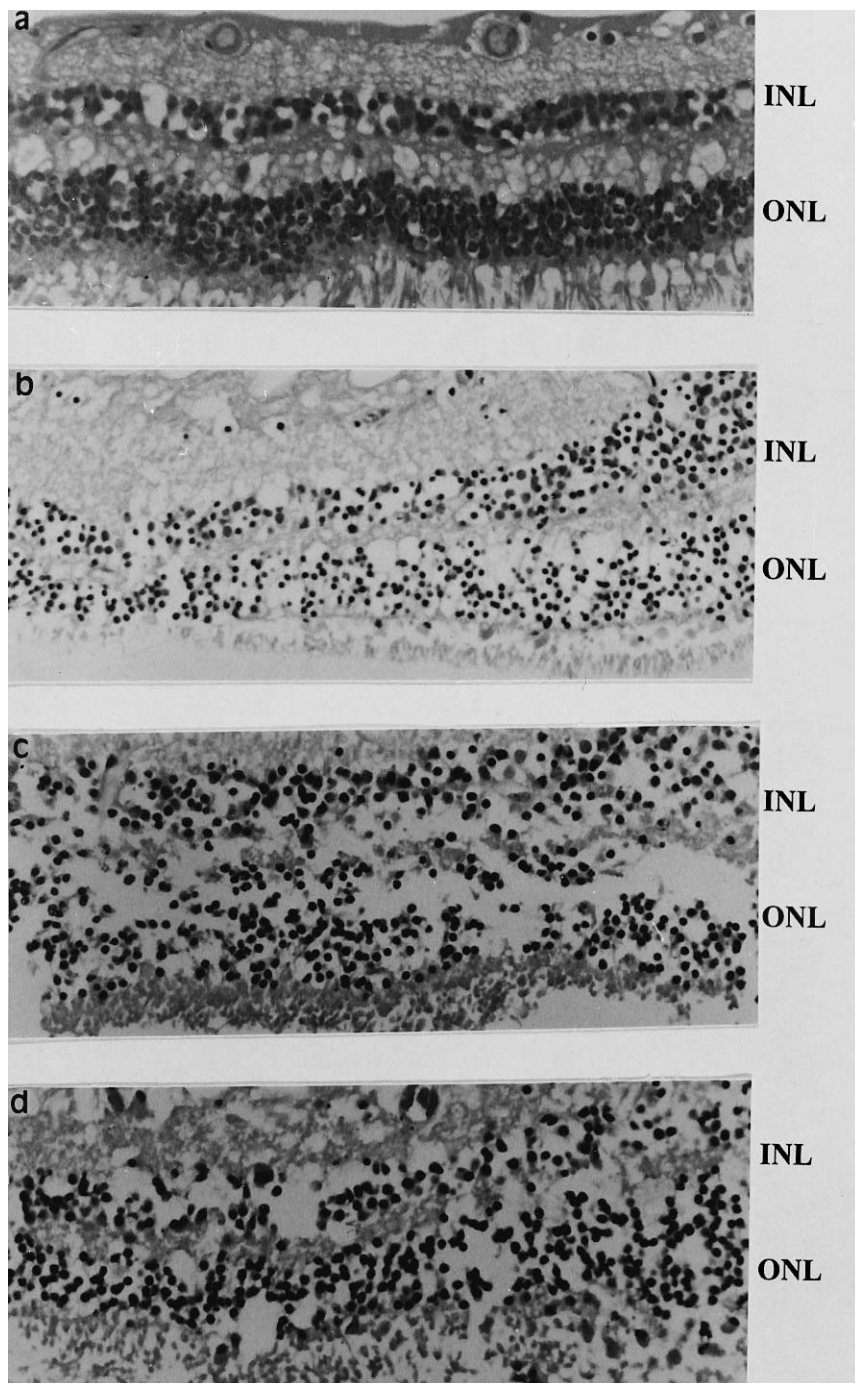

Fig.4a-d Cross section through human retinal explants stained with haemotoxylin and eosin. (a) Photomicrographs of retinal explant sections prior to culture and after (b) $1,(\mathbf{c})$ 3 and (d) 10 days in culture within a fibrin matrix. The location of the inner nuclear layer (INL) and the outer nuclear layer $(\mathrm{ONL})$ is indicated (mag. $\times 600)$

showed evidence of disintegration of the retinal architecture that progressed to show further degeneration after $3(6 / 6)$ and 10 days (6/6) in culture (Fig. 4) as determined by $\mathrm{H} \& \mathrm{E}$ staining.

Immunopositivity to the vWF antibody indicated an increase in the number and the size of vessels within the inner nuclear layer after only $24 \mathrm{~h}$ in culture which developed further in the inner nuclear layer (Fig. 5a-d). By day 10, an extensive vascular network was evident both within the retina and extending out from the periphery of the explant into the surrounding matrix.

Weak immunoreactivity to GFAP was evident in the retina prior to culture (Fig. 5 e). We found GFAP associated with retinal vessels within $24 \mathrm{~h}$ of culture (Fig. 5f) and in particular was closely associated with 

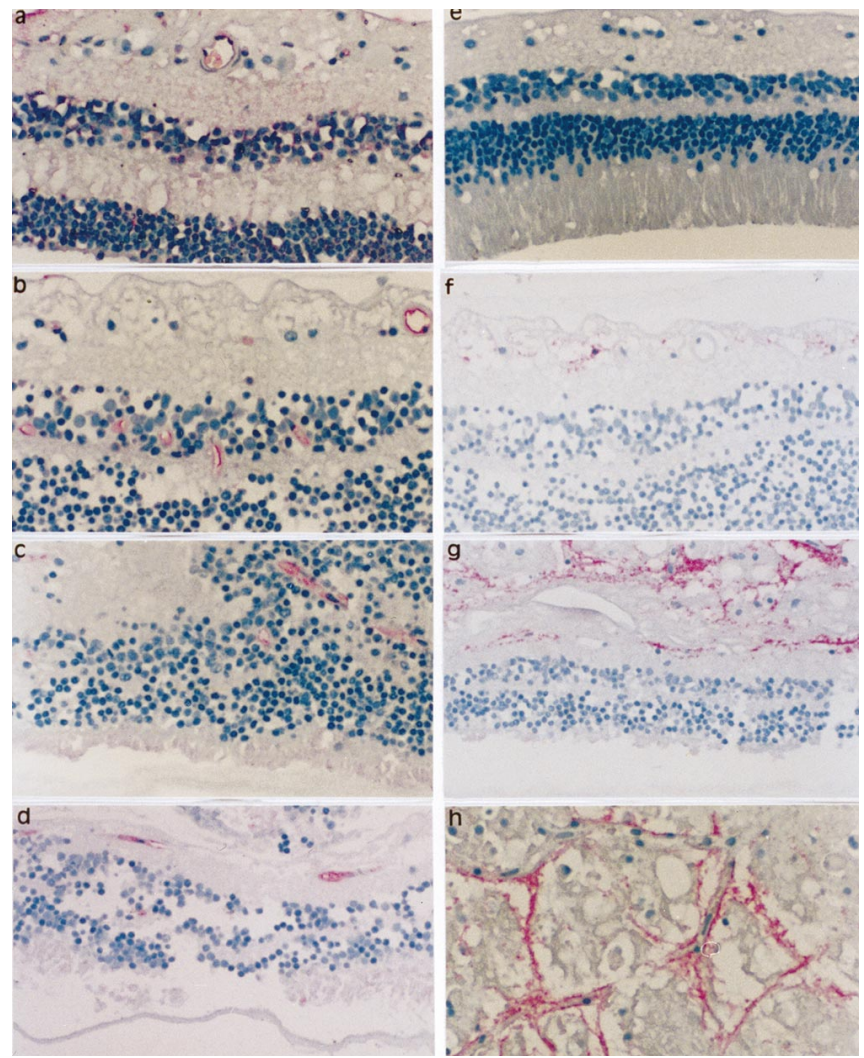

Fig.5 a-h Increased expression of von Willebrand's factor and glial fibrillary acidic protein in human retinal explants. Von Willebrand immunoreactivity in a human retina prior to culture (a) is compared to the increase in immunoreactivity to vWF staining after (b) 1, (c) 3 and (d) 10 days in culture. Prior to culture, human retina showed weak immunostaining for GFAP (e). With increasing duration of culture at day 1 (f) and 3 (g), the explants showed evidence of increased staining associated with the ganglion cell layer. By day $10(\mathbf{h})$, this was strongly associated with the developing network of vessels that was extending from the periphery of the explant and associated with the vessel wall located within the outer nuclear layer of the explant $(\mathbf{a}-\mathbf{g}$ mag. $\times 600 ; \mathbf{h}$ mag. $\times 1200)$

the new network of vessels that was evident by day 10 (Fig. 5 g,h). The intensity of staining for GFAP increased with the duration of culture.

Evidence for monocyte activation associated with retinal angiogenesis. Cells positive for CD68 were identified within 3 days of culture within retinal vessels (Fig. 6a), closely associated with the vessel (Fig. 6b) and after migration within the body of the retina (Fig. 6c) and were detected up to day 15. Positive immunoreactivity within the vessels is due to macrophages whereas that evident within the body of the retina is due to either macrophages or microglia. Positive immunoreactivity to Mib1 was evident in cells that were morphologically identical to CD68 positive cells in serial sections from the same explant (Fig. 6d) and were found closely associated with endothelial cells stained positively with Mib1 (Fig.6e).
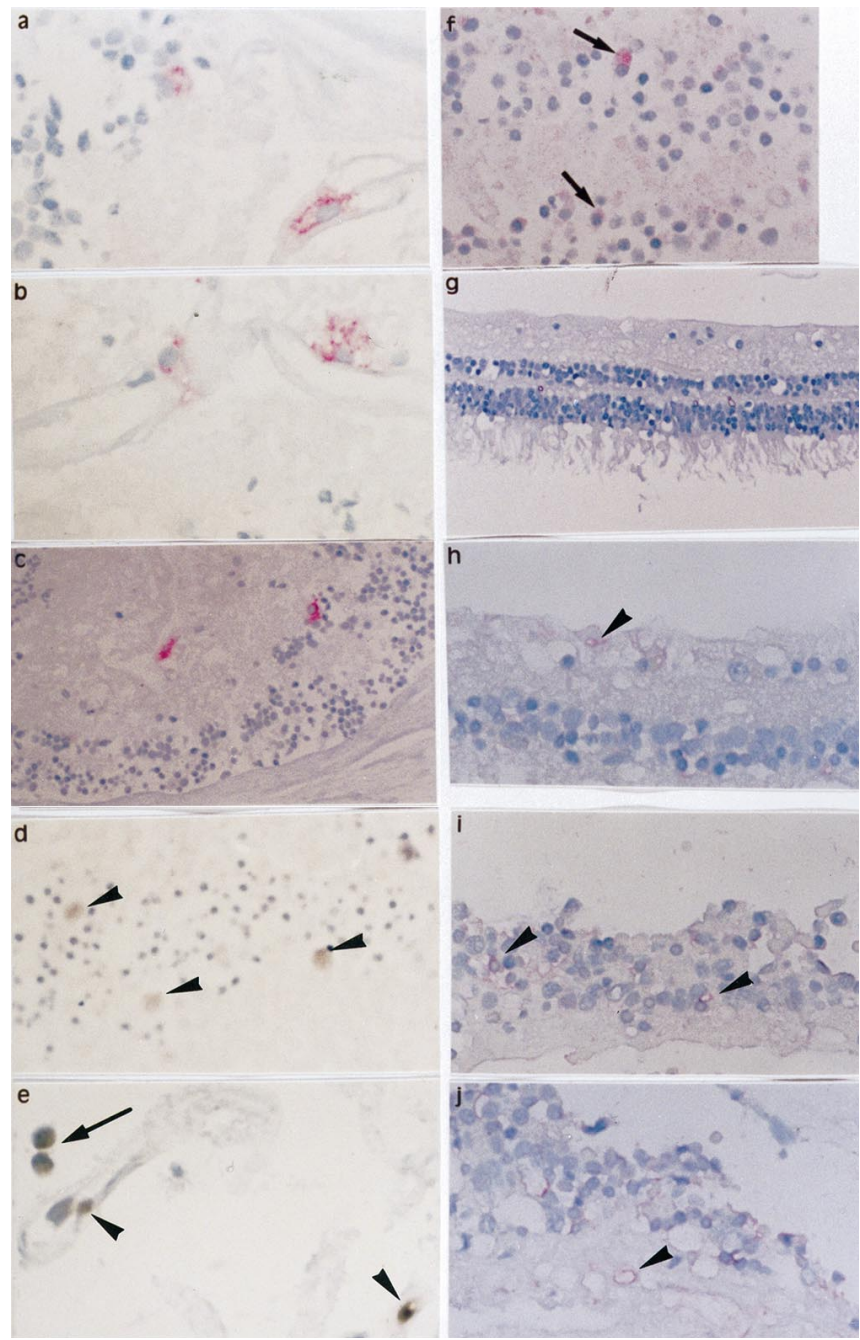

Fig.6a-j Involvement of macrophages, VEGF and MCT-1 in human retinal vessel growth. CD68 positive cells were found in sections from human retinal explants from day 3 within the vessels (a), closely associated with the vessel wall (b) $(\mathrm{mag} . \times 1200)$ or within the body of the retina (c) (mag. $\times 600)$. Positive immuoreactivity to the Ki-67 antigen was evident in cells that were morphologically similar and located in similar areas to CD68 positive cells $(\mathbf{d})(\mathrm{mag} . \times 600)$. Positive staining for $\mathrm{Ki}-67$ was also associated with retinal vessels (arrow) (e) (mag. $\times 1200)$. Mib1 positive nucleated macrophage-like cells were also found closely associated with the vessel (e). Positive immunoreactivity to VEGF antibody (f) (mag. $\times 1200)$ indicated that VEGF was being produced endogenously by the retinal cells. No positive immunoreactivity to MCT-1 was evident in the retina prior to culture (g) or in the absence of exogenous VEGF (mag. $\times 520$ ). In explants which had VEGF supplemented to the growth medium and the matrix MCT-1 was detected (h-j), (h and i mag. $\times 600$; j Mag. $\times 1050)$ at day 3 post-culture

Vascular endothelial growth factor-mediated increased expression of monocarboxylate transporter type 1. Localised expression of endogenous VEGF was evident (Fig. 6f) after 3 days of culture. Immunohistochemistry was unable to detect the MCT-1 in the retina prior to culture (Fig. 6g). After the explants were cultured 
in the presence of exogenous VEGF, positive immunoreactivity to MCT-1 was, however, evident. In the absence of VEGF, no MCT-1 specific staining was evident.

\section{Discussion}

We present details of a model system for human retinal angiogenesis. Furthermore, we describe evidence for the activation of both endothelial cells and monocytes and the increased expression of GFAP associated with new vessel growth. We also show that MCT-1 is expressed in the retinal endothelium of the explant and is absent in the retina prior to culture.

One of the main advantages of the human retinal explant model is that it is possible to maintain retinal vessel architecture and to assess the contribution that other cell types make to the growth of new vessels. Previous work with the bovine retina [28] has shown that the ischaemic retinal explant promotes new vessel growth by providing an environment that promotes the survival of endothelial cells and macrophages. We now confirm that macrophages or microglia cells or both are associated with endothelial cell proliferation within the human retinal explant. Thus, either macrophages extravasate into the surrounding retinal tissue or the presence of macrophages within the vessels is associated with activation of resident micoglia which at their end stage resemble macrophages [30].

Leucocyte activation is evident in animal models of diabetes $[9,10]$ and in human leucocytes [11] with an association of monocytes with neovascularisation $[10,31]$. Furthermore, there is evidence for endothelial activation in diabetes, as seen by increased soluble E selectin in serum [8] and this provides an environment whereby monocyte adhesion is promoted. Capillary occlusion may result from the physical 'plugging' of the capillary and the resulting ischaemia may activate growth factors that are required to initiate new vessel growth. Alternatively, or possibly in conjunction with the plugging of the vessels is the action of monocyte derived growth factors that are released from the adherent cell. Interestingly, monocyte derived growth factors have been shown to be essential for the growth of endothelial cells in vitro [6] and it is likely that a combination of these factors is important.

A number of potential mechanisms possibly occur within the retinal explant that explain the new vessel growth and the activation of monocytes. The increased expression of VEGF mRNA by rat vascular endothelial cells [32] and VEGF mRNA and protein in retinal glial cells [5] in hypoxic conditions provides a source of endothelial mitogen. The expression of VEGF in the human retina was confirmed in this study. Paradoxically, hypoxia has been shown to re- sult in a reduced expression of fibroblast growth factors [7], although monocytes will secrete both bFGF and PDGF in response to hypoxia which can increase endothelial cell proliferation [6]. The localised production of growth factors and cytokines by mononuclear cells therefore provides a sustainable source of factors which have the potential to act as chemoattractants for other mononuclear cells, and also for promotion of adhesion to the retinal endothelium.

Increased expression of GFAP has been shown to be increased in the retina after 3 months of experimentally induced diabetes and was also associated with impaired glial glutamate activity [15]. Increased expression of GFAP is also evident in this study and was found associated with new vessel growth. This substantiates existing evidence for the contribution of glial cell function in retinal damage and we can now confirm that a change in GFAP immunoreactivity is evident in the human retina that reflects the progressive retinal dysfunction with increasing duration of culture. Other factors relating to the matrix components and the factors that influence the growth of vessels would be possible with this system and may prove invaluable in determining the mechanism of retinal angiogenesis.

Poor glucose control is known to increase the severity and incidence of the complications of diabetes which is also influenced by the duration of diabetes [20]. In this study we were not able to detect any effect of glucose concentration on vessel growth although the duration of exposure to high concentrations of glucose was relatively short. An observable effect of glucose concentration in this model system is perhaps evident with a more prolonged exposure to high concentrations of glucose or the presence of glycated matrix or both [33].

Retinal ischaemia is a characteristic feature of diabetic retinopathy [34] and glucose metabolism in these conditions will generate high concentrations of lactate. In the retinal explant model described here the absence of blood flow and the tissue culture conditions in themselves maintain a relative hypoxia [28]. Therefore we examined the expression of MCT-1 in the retinas and found it to be expressed post-culture. Failure to detect MCT- 1 in the retinas prior to culture lends credence to the theory that the expression of this transporter is associated with the hypoxia of the explants. The excess production of lactate may result in an imbalance of NAD/NADH leading to oxidative mediated microvascular damage. This may further enhance the potential effects by the already reduced anti-oxidative reserve in patients with diabetes [35] and account for the improved outcome of ischaemia / re-perfusion injuries with antioxidant therapy [36]. Another requirement for the detection of the MCT-1 was, however, the presence of exogenous VEGF which is itself shown to be associated with hypoxia $[37,38]$. The extremely high meta- 
bolic rate of the retina exacerbates the potential impact that any change in glucose availability may have. Thus, the combined effects of ischaemia, fluctuating glucose concentrations and the high energy demands of the retina, uniquely predispose this tissue to damage.

A model system for human retinal angiogenesis is invaluable in the study of diabetic retinopathy and has the potential for wide application. In this study we have shown how this model can be used to show specific key components of retinal angiogenesis.

Acknowledgements. We would like to thank The Wellcome Trust, and the Aberdeen Royal Hospital Trust Endowment Fund for the financial support of this work. We would also like to thank the members of the Department of Pathology at the University of Aberdeen for their invaluable assistance in the embedding, sectioning and $\mathrm{H} \& \mathrm{E}$ staining of the retinal explants. We are indebted to Dr. L. Pels of the National Ophthalmic Research Institute of the Netherlands, Amsterdam for the support of this research.

\section{References}

1. Forrester JV, Knott RM (1997) Pathogenesis of diabetic retinopathy and cataract. In: Pickup JC, Williams G (eds) Textbook of diabetes. Blackwell Science, Oxford, pp 451-470

2. Barinaga M (1995) Shedding light on blindness. Science 267: 452-453

3. Hudlika O, Eggington S, Dawson JM (1992) Effect of ischaemia on endothelial cells. In: Maragoudakis ME, Gullino P, Lelkes PI (eds) Angiogenesis in health and disease. Plenum Press, New York, London, pp 143-147

4. Tischer E, Gospodarowicz D, Mitchell PJ et al. (1989) Vascular endothelial growth factor: a new member of the platelet-derived growth factor gene family. Biochem Biophys Res Commun 165: 1198-1206

5. Murata T, Nakagawa K, Khalil A et al. (1996) The relation between expression of vascular endothelial growth factor and breakdown of the blood-retinal barrier in diabetic rat retinas. Lab Invest 74: 819-825

6. Kuwbara K, Ogawa S, Matsumoto M et al. (1995) Hypoxiamediated induction of acidic/basic fibroblast growth factor and platelet-derived growth factor in mononuclear phagocytes stimulates growth of hypoxic endothelial cells. Proc Natl Acad Sci 92: 4606-4610

7. Grant MM, Koo H-C, Rosenfeld MG (1992) Oxygen affects human endothelial cell proliferation by inactivation of fibroblast growth factors. Am J Physiol 263: L370-L375

8. Olson JA, Whitelaw CM, McHardy KC, Pearson DWM, Forrester JV (1997) Soluble leucocyte adhesion molecules in diabetic retinopathy stimulate retinal capillary endothelial cell migration. Diabetologia 40: 1166-1171

9. Freedman SF, Hatchell DL (1992) Enhanced superoxide radical production by stimulated polymorphonuclear leucocytes in a cat model of diabetes. Exp Eye Res 55: 767-773

10. Schroder S, Palinski W, Schmid-Schonbein GW (1991) Activated monocytes and granulocytes, capillary nonperfusion and neovascularization in diabetic retinopathy. Am J Pathol 139: 81-100

11. Simmons D, Ng LL, Bomford J (1993) Abnormal myoinositol influx in human leucocytes in diabetes but not specifically in diabetic neuropathy. Diabetes 41: 760-765
12. Arnould T, Michiels C, Remacle J (1993) Increased PMN adherence on endothelial cells after hypoxia: involvement of PAF, CD18/CD11b, and ICAM-1. Am J Physiol 264: C1102-C1110

13. Adamis AP, Shima DT, Tolentino MJ et al. (1996) Inhibition of vascular endothelial growth factor prevents retinal ischemia-associated iris neovascularization in a nonhuman primate. Arch Ophthalmol 114: 66-71

14. Amin RH, Frank RN, Kennedy A et al. (1997) Vascular endothelial growth factor is present in glial cells of the retina and optic nerve of human subjects with nonproliferative diabetic retinopathy. Invest Ophthalmol Vis Sci 38: 36-47

15. Lieth E, Barber AJ, Xu B et al. (1998) Glial reactivity and impaired glutamate metabolism in short-term experimental diabetic retinopathy. Diabetes 47: 815-820

16. Roy M, Gunkel R, Podgor M (1986) Colour vision defects in early diabetic retinopathy. Arch Ophthalmol 104: 225-228

17. Sokol S, Moskowitz A, Skarf B (1985) Contrast sensitivity in diabetics with and without background retinopathy. Arch Ophthalmol 103: 51-54

18. Newman E, Reichenbach A (1996) The Muller cell: a functional element of the retina. Trends Neurosci 19: 307-312

19. Ohira A, De JJ (1990) Characterization of glial involvement in proliferative diabetic retinopathy. Ophthalmologica 201: 187-195

20. The Diabetes control of complications trial. (1993) The effect of intensive treatment of diabetes on the development and progression of long-term complications in insulin-dependent diabetes mellitus. N Engl J Med 329: 977-986

21. Kumagai AK, Glasgow BJ, Pardridge WM (1994) GLUT1 glucose transporter expression in the diabetic and nondiabetic human eye. Invest Ophthalmol Vis Sci 35: 2887-2894

22. Knott RM, Robertson M, Muckersie E, Forrester JV (1996) Regulation of glucose transporters (GLUT-1 and GLUT-3) in human retinal endothelial cells. Biochem J 318: 313-317

23. Vilchis C, Salceda R (1996) Characterization of $[2-3 \mathrm{H}]$ deoxy-D-glucose uptake in retina and retinal pigment epithelium of normal and diabetic rats. Neurochem Int 28: 213-219

24. Poole RC, Halestrap AP (1993) Transport of lactate and other monocarboxylates across mammalian plasma membranes. Am J Physiol 264: C761-C782

25. Price NT, Jackson VN, Halestrap AP (1998) Cloning and sequencing of four new mammalian monocarboxylate transporter (MCT) homologues confirms the existence of a transporter family with an ancient past. Biochem J 329: 321-328

26. Baker SK, McCullagh KJA, Bonen A (1998) Training intensity-dependent and tissue-specific increases in lactate uptake and MCT-1 in heart and muscle. Journal of Applied Physiology: Respiratory, Environ Exerc Physiol 84: 987-994

27. Broer S, Rahman B, Pellegri G et al. (1997) Comparison of lactate transport in astroglial cells and monocarboxylate transporter 1 (MCT 1) expressing Xenopus laevis oocytes. Expression of two different monocarboxylate transporters in astroglial cells and neurons. J Biol Chem 272: 30096-30102

28. Forrester JV, Chapman A, Kerr C et al. (1990) Bovine retinal explants cultured in collagen gels. Arch Ophthalmol 108: 415-420

29. Cordell JL, Falini B, Erber WN et al. (1984) Immunohistochemical labelling of monoclonal antibodies using immune complexes of alkaline phosphatase and monoclonal anti-alkaline phosphatases (APAAP complexes). J Histochem Cytochem 32: 219-229 
30. Kreutzberg GW (1996) Microglia: a sensor for pathological events of the CNS. Trends in Neuro Sci 19: 312-318

31. Arras M, Ito WD, Scholz D et al. (1998) Monocyte activation in angiogenesis and collateral growth in the rabbit hindlimb. J Clin Invest 101: 40-50

32. Levy AP, Levy NS, Wegner S, Goldberg MA (1995) Transcriptional regulation of the rat vascular endothelial growth factor gene by hypoxia. J Biol Chem 270: 13333-13340

33. Kuzuya M, Satake S, Ai S et al. (1998) Inhibition of angiogenesis on glycated collagen lattices. Diabetologia 41: 491-499

34. D'Amore PA (1994) Mechanisms of retinal and choroidal neovascularization. Invest Ophthalmol Vis Sci 35: 3974-3978
35. Barnett AH (1993) Origin of the microangiopathic changes in diabetes. Eye 7: 218-222

36. Roy S, Sen CK, Tritschler HJ, Packer L (1997) Modulation of cellular reducing equivalent homeostasis by alpha-lipoic acid. Mechanisms and implications for diabetes and ischemic injury. Biochem Pharmacol 53: 393-399

37. Forsythe JA, Jiang B-H, Iyer NV et al. (1996) Activation of vascular endothelial growth factor gene transcription by hypoxia-inducible factor 1. Mol Cell Biol 16: 4604-4613

38. Levy AP, Levy NS, Wegner S, Goldberg MA (1995) Transcriptional regulation of the rat vascular endothelial growth factor gene by hypoxia. J Biol Chem 270: 13333-13340 